\title{
The Characteristic of Thermoelastic Waves in Transversely Isotropic Finite Cylinders
}

\author{
Hao Bai \\ Lakehead University, Ontario, Canada, P7B5E1
}

\begin{abstract}
A theoretical as well as a numerical investigation of the propagation of thermoelastic waves and vibration of transversely isotropic cylinders of finite length is discussed. Lord-Shulman theory is adopted here to model the thermoelastic deformation of cylinders. A semi analytical finite element (SAFE) method is employed to study dispersion of thermoelastic waves and natural frequencies of vibration of finite cylinders with traction free curved surfaces having both ends insulated and constrained by frictionless rigid walls. Numerical results obtained by the SAFE method for the frequencies of vibration of a sapphire rod are found to be in excellent agreement with published results. Natural frequencies of vibration for first three axisymmetric and asymmetric modes are presented graphically for a silicon nitride thermoelastic cylinder. Also, numerical results showing dispersion of both propagating and evanescent circumferential waves in infinite and finite cylinders are presented also.
\end{abstract}

\section{Introduction}

Isothermal vibration of finite-length cylinders has been studied by many investigators. Since exact analytical solutions for a finite-length vibrating cylinder cannot be obtained in general, approximate solutions have been proposed to estimate natural frequencies of finite isotropic circular cylinders by [1]-[5]. If the material of the cylinder is anisotropic, the solution of the free vibration problem becomes more complex. [6] used separation of variables technique presented by [7], who derived the dispersion equations governing compressional wave propagation along the axis of a transversely isotropic cylinder. In their study [6], they considered the axisymmetric vibrations of finite - length transversely isotropic cylinders and presented experimental results for sapphire rods. [8] estimated natural frequencies of axisymmetric modes of finite length transversely isotropic cylinders by applying the Ritz method. [9] and [10] studied axisymmetric and asymmetric vibrations of a finite transversely isotropic cylinder using potential functions and compared their results with the experimental observations of [6]. [11] studied free vibrations of finite orthotropic thin cylindrical shells by employing shell theory.

In this paper, thermoelastic circumferential wave propagation in a finite - length transversely isotropic cylinder has been investigated. [12] studied analytically the two-dimension problem of isothermal guided circumferential waves in a circular annulus. To our knowledge, propagating and evanescent modes of circumferential waves in a finite-length transversely isotropic cylinder have not been studied in the past. Since finite - length cylinders are common structural components in many practical applications, the safety of these structural components must be assessed regularly for their continual usage or for their repair. In order to monitor their health, it is necessary to assess their material properties and detect critical cracks or defects in them. Guided ultrasonic waves have proven to be very efficient for structural health monitoring (SHM). In recent years, laser based ultrasonic techniques for material property measurements and defect characterizations have proven to be very reliable. The coupled theory of thermoelasticity must be used to find complete solutions for the guided waves generated by pulsed lasers.

Recently the thermoelastic wave in a generalized cross section cylinder immersed in a fluid is studied by using the Fourier expansion collocation method by [13]. [14] studied the propagation of thermoelastic circumferential waves in orthotropic cylindrical curved plates using the Green-Naghdi (GN) generalized thermoelastic theory (without energy dissipation). It is shown that thermal properties have no effect on the circumerential SH wave and the thermoelasticity has very limited effects on the other circumferential modes. In 2011, a work on the randomness of thermoelasticity wave in thick hollowed cylinder was reported [15]. They analytically studied the thermal and mechanical waves using theory of coupled thermoelasticity without energy dissipationbased on Green-Naghdi model.

Propagation of thermoelastic waves in a circular annulus and finite length circular cylindrical shells have been studied here in the context of LS [16] generalized theory of thermoelasticity. A semi-analytical finite 
element (SAFE) method [17-19, and references therein] is employed to study characteristics of guided wave modes in the cylinder. The dispersion equation governing the modes is obtained in the form of an eigenvalue problem. Solutions obtained by the SAFE formulation are validated by comparing the numerical results for finite length transversely isotropic elastic cylinders (isothermal case) with those published by [9], [10]. Frequency spectra obtained for the (plane strain) circumferential wave propagation problem are shown to agree well with those published by [12] for an isotropic (isothermal) case. Numerical results are presented here for both propagating and evanescent modes in a transversely isotropic silicon nitride cylinder.

\section{Equations of motion}

Consider a finite - length transversely isotropic circular cylinder of inner radius $r_{i}$ and outer radius $r_{o}$ in the cylindrical coordinate system $(r, \theta, z)$ shown in Fig. 1. The cylinder is initially in the undisturbed state with uniform temperature $T_{0}$. The inner and outer surfaces of the cylinder are traction free and the heat flux vanishes at inner and outer surfaces in the radial direction. The ends of the cylinder are insulated thermally and also constrained by frictionless rigid walls.
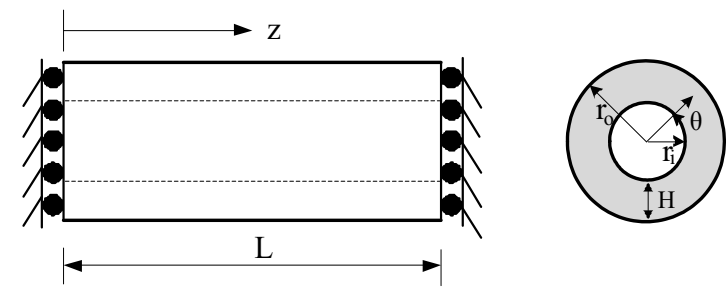

Figure 1. Geometry of the cylinder

The generalized LS governing equations of thermoelasticity, in the absence of body force and heat source, are given by [20] as,

$$
\begin{gathered}
\sigma_{i j, j}=\rho \ddot{u}_{i} \\
T_{0} \rho \dot{\eta}=-q_{i, i} \\
\sigma_{i j}=C_{i j k l} \varepsilon_{k l}-\beta_{i j} T \\
\rho \dot{\eta}=\beta_{i j} \dot{\varepsilon}_{i j}+\frac{\rho c_{E}}{T_{0}} \dot{T} \\
q_{i}+\tau_{0} \dot{q}_{i}=-k_{i j} T_{, j}
\end{gathered}
$$

here $\sigma_{i j}$, the components of the stress tensor; $u_{i}$, the components of the displacement; $\varepsilon_{i j}$, the components of the strain tensor; $C_{i j k l}$, the elastic constants; $q_{i}$, components of the heat flux vector; $\rho$, the mass density; $T_{0}$, the reference temperature; $\eta$, the entropy density; $T$, the temperature change; $c_{E}$, the specific heat at constant deformation; $\tau_{0}$, the thermal relaxation time; $\beta_{i j}$, the thermal coefficients; $k_{i j}$, the coefficients of thermal conductivity. In the above equations, the superposed dot indicates the derivative with respect to time.

A normalization procedure is needed to find the solution of the problem. A careful identified set of basic variables was choosen and see detail in [21]. It may be noted that the non-dimensional scheme yields all dimensionless equations in the same form as their dimensional counterparts. Therefore, this normalization can be used to solve multilayer cylinders as well. The final equations of motion in terms of displacements and temperature are given by

$$
\begin{gathered}
c_{i j k l} u_{k, l j}-\beta_{i j} T_{, j}=\rho \ddot{u}_{i} \\
\beta_{i j} T_{0}\left(1+\tau_{0} \frac{\partial}{\partial t}\right) \dot{u}_{i, j}-k_{i j} T_{i j}+\rho c_{E}\left(1+\tau_{0} \frac{\partial}{\partial t}\right) \dot{T}=0
\end{gathered}
$$

It's worth to mention that the above quantities are in their respective non-dimensional form.

\section{Semi analytical FE formulation}

In this method, for a finite cylinder of length $\mathrm{L}$, the radial dependence of the displacement, $\mathbf{u}$, and temperature, $\mathrm{T}$, is approximated by one dimensional isoparametric finite elements. The total thickness of the cylinder, $H$, is composed of cylindrical layers and each layer can have distinct thermoelastic properties and thickness. By using the SAFE method, thickness of the cylinder is discretized into $N$ laminas. Quadratic interpolation polynomials are used to approximate the displacement and temperature field over each lamina in the radial direction.

The displacements and temperature of the kth lamina are expressed as

$$
\begin{aligned}
& \mathbf{u}(r, \theta, z, t)=Z \mathbf{N}_{1}(\zeta) \mathbf{u}^{e}(\theta, t) \\
& \mathbf{T}(r, \theta, z, t)=c \mathbf{N}_{2}(\zeta) \mathbf{T}^{e}(\theta, t)
\end{aligned}
$$

and

here

$$
\begin{aligned}
& \mathbf{N}_{1}(\zeta)=\left[\begin{array}{ccccccccc}
n_{1} & 0 & 0 & n_{2} & 0 & 0 & n_{3} & 0 & 0 \\
0 & n_{1} & 0 & 0 & n_{2} & 0 & 0 & n_{3} & 0 \\
0 & 0 & n_{1} & 0 & 0 & n_{2} & 0 & 0 & n_{3}
\end{array}\right], \\
& \mathbf{N}_{2}(\zeta)=\left[\begin{array}{lll}
n_{1} & n_{2} & n_{3}
\end{array}\right], \quad \mathbf{Z}=\left[\begin{array}{ccc}
c & 0 & 0 \\
0 & c & 0 \\
0 & 0 & s
\end{array}\right] \\
& \mathbf{u}^{e}=\left(u_{r 1}, u_{\theta 1}, u_{z 1}, u_{r 2}, u_{\theta 2}, u_{z 2}, u_{r 3}, u_{\theta 3}, u_{z 3}\right)^{T}, \\
& \mathbf{T}^{e}=\left(T_{1}, T_{2}, T_{3}\right)^{T}, \mathbf{R}^{e}=\left(R_{b}, R_{m}, R_{f}\right)^{T}
\end{aligned}
$$

where $s=\sin (p z), c=\cos (p z), p=m \pi / L$ and $L$ is the length of the cylinder and, $m$ is the axial wave number. The shape functions are 


$$
\begin{aligned}
& n_{1}=\frac{1}{2} \zeta(\zeta-1), \\
& n_{2}=1-\zeta^{2}, \\
& n_{3}=\frac{1}{2} \zeta(\zeta+1), \quad-1 \leq \zeta \leq 1
\end{aligned}
$$

where

$$
\zeta=\frac{2 r-R_{b}-R_{f}}{R_{f}-R_{b}} \quad\left(R_{b} \leq r \leq R_{f}\right)
$$

The nodal displacements $u_{r j}, u_{\theta j}, u_{z j}$, and temperature $T_{j}$, where $\mathrm{j}=1,2,3$, are taken at the inner surface $R_{b}$, middle layer $R_{m}$ and the outer surface $R_{f}$ of the $k^{\text {th }}$ lamina.

By employing the variational principle of thermoelasticity [20], the equations of motion can be written as, in the matrix form,

$$
\mathbf{E}_{1} \ddot{\mathbf{V}}+\mathbf{E}_{2} \ddot{\mathbf{V}}_{, \theta}+\mathbf{E}_{3} \dot{\mathbf{V}}+\mathbf{E}_{4} \dot{\mathbf{V}}_{, \theta}+\mathbf{E}_{5} \mathbf{V}+\mathbf{E}_{6} \mathbf{V}_{, \theta}+\mathbf{E}_{7} \mathbf{V}_{, \theta \theta}=0
$$

where $\mathbf{E}_{i}(i=1,2, \ldots, 7)$ are the global matrices, see details in [20], $\mathbf{V}$ is the global nodal displacement and temperature vector.

Traction free boundary conditions on surfaces of cylinder require that the stresses at inner and outer surfaces of the cylinder are zero

$$
\sigma_{r r}=\sigma_{r \theta}=\sigma_{r z}=0 \quad \text { at } \quad r=r_{i} \text { and } r=r_{o}
$$

End boundary conditions of the cylinder are

$$
u_{z}=\sigma_{r z}=\sigma_{\theta z}=0 \quad \text { at } \quad z=0, L
$$

Thermal boundary conditions are considered as

$$
\begin{array}{lll}
T,_{z}=0 & \text { at } & z=0, L \\
T_{, r}=0 & \text { at } & r=r_{i} \text { and } r=r_{o}
\end{array}
$$

The solution of Eq. (11) is assumed as

$$
\mathbf{V}=\mathbf{V}_{0} e^{i(n \theta-\omega t)}
$$

where, $\mathbf{V}_{0}$ is the nodal amplitude vector and $\omega$ is the nondimensional circular frequency. For waves propagating in the $\theta$-direction, $e^{i n \theta}$ is defined as $e^{i n \theta}=e^{i\left(k r_{o}\right) \theta}$ where $k$ is the wave number defined as $k=\omega / c\left(r_{o}\right) . c(r)$ is the phase velocity of the circumferential wave travelling along the surface at a distance $r$. Substitution of Eq. (12) into (11) results in a set of linear homogeneous equations. For integer values of $m$, two eigenvalue problems can be obtained depending on whether $n$ or $\omega$ is chosen as the eigenvalue. If $n$ serves as the eigenvalue with assigned values for $\omega$, it will give a quadratic eigenvalue problem:

$$
\begin{aligned}
& \left(-n^{2} \mathbf{E}_{7}+n \mathbf{E}_{B}+\mathbf{E}_{A}\right) \mathbf{V}_{0}=0 \\
\mathbf{E}_{A}= & \left(-\omega^{2} \mathbf{E}_{1}-i \omega \mathbf{E}_{3}+\mathbf{E}_{5}\right) \\
\mathbf{E}_{B}= & \left(-\omega^{2} i \mathbf{E}_{2}+\omega \mathbf{E}_{4}+i \mathbf{E}_{6}\right)
\end{aligned}
$$

Equation (13) can be converted into a first order equation in the form

$$
\left[\begin{array}{cc}
0 & \mathbf{I} \\
\mathbf{E}_{A} & \mathbf{E}_{B}
\end{array}\right]\left\{\begin{array}{c}
\mathbf{V}_{0} \\
n \mathbf{V}_{0}
\end{array}\right\}=n\left[\begin{array}{cc}
\mathbf{I} & 0 \\
0 & \mathbf{E}_{7}
\end{array}\right]\left\{\begin{array}{c}
\mathbf{V}_{0} \\
n \mathbf{V}_{0}
\end{array}\right\}
$$

Solution of the generalized eigenvalue problem (14) gives the dispersion relation for guided circumferential thermoelastic waves in the finite cylinder. On the other hand, for given integer values of $m$ and $n$, i twill generate the frequencies of free thermoelastic vibration of the shell, see detail in [21].

\section{Numerical results and discussion}

In order to validate the SAFE formulation, a couple of examples were studied and results were compared with published literatures. The first example was guided circumferential waves for the isothermal plane strain problem, it was reported in [12]. The comparison between SAFE results and those obtained by Liu and $\mathrm{Qu}$ [12] is shown in below in Fig. 2. The agreement is excellent. The second example was the vibration of a finite transversely isotropic cylinder and it was compared with the results in $[9,10]$, the comparison does not show here to save space. They all showed excellent agreement.

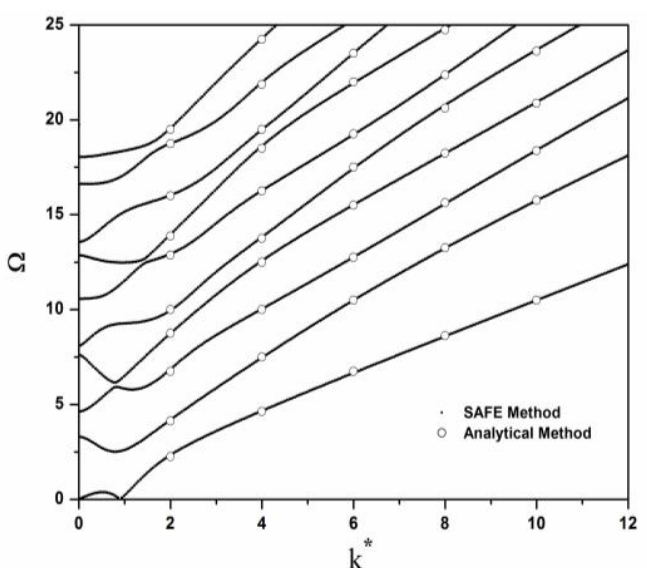

Figure 2. 2D view of propagating modes for $\eta_{s}=0.1$ ([12], Fig. 2(a))

A finite cylindrical tube of length $15 \mathrm{~mm}$ with inner and outer radii of $1.5 \mathrm{~mm}$ and $2.5 \mathrm{~mm}$, respectively, is considered here. Thus, the nondimensional length is 15 and, nondimensional inner and outer radii are, respectively, $r_{i}=1.5$ and $r_{o}=2.5$. The material of the tube is taken to be transversely isotropic silicon nitride $\left(\mathrm{Si}_{3} \mathrm{~N}_{4}\right)$ [20]. The symmetry axis of the material is aligned with the axis of the cylinder. For the purpose of nondimensionalization, normalizing velocity $\bar{v}$ is chosen to be $0.25 \mathrm{~km} / \mathrm{s}$. Furthermore, the normalized density $\rho^{*}$ is taken to be 1 . Then, the nondimensional material properties of the tube are given by

$$
\begin{aligned}
& T_{0}^{*}=1.0, \quad \tau_{0}^{*}=1.081 \times 10^{-7}, \quad \beta_{r r}^{*}=\beta_{\theta \theta}^{*}=70.03, \quad \beta_{z z}^{*}=83.21 \\
& k_{r r}^{*}=k_{\theta \theta}^{*}=0.0785, \quad k_{z z}^{*}=0.10, c_{E}^{*}=967.50, \quad c_{11}^{*}=\frac{c_{11}}{\bar{c}}=2165, \\
& c_{12}^{*}=\frac{c_{12}}{\bar{c}}=975, c_{13}^{*}=\frac{c_{13}}{\bar{c}}=635, c_{33}^{*}=\frac{c_{33}}{\bar{c}}=2870, c_{44}^{*}=\frac{c_{44}}{\bar{c}}=540
\end{aligned}
$$


The dispersion curves for thermoelastic waves in a cylinder of finite length were evaluated for different value of $m$ - the wave number in the axial direction. Group velocities of the circumferential waves are computed at the outer surface of the cylinder as well. Due to the limitation, only the results for $m=1$ are presented here.

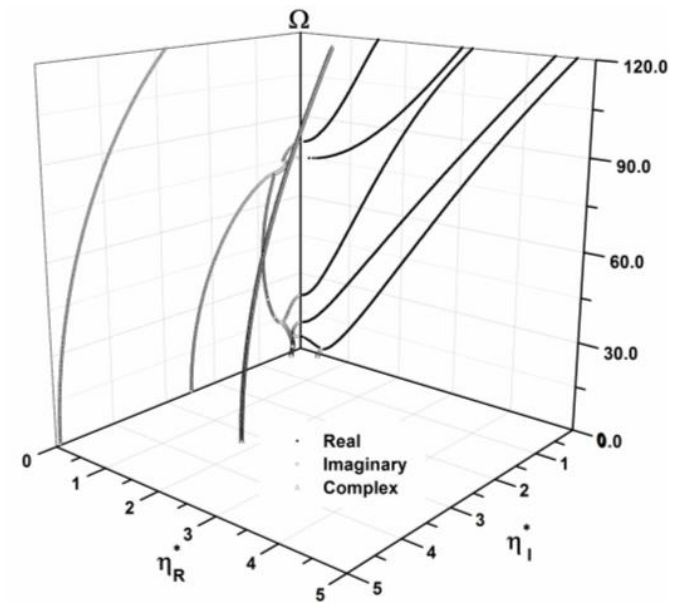

Figure 3. 3D view of frequency - circumferential wave number plots of a silicon nitride cylinder when axial wave number, $m=$ 1

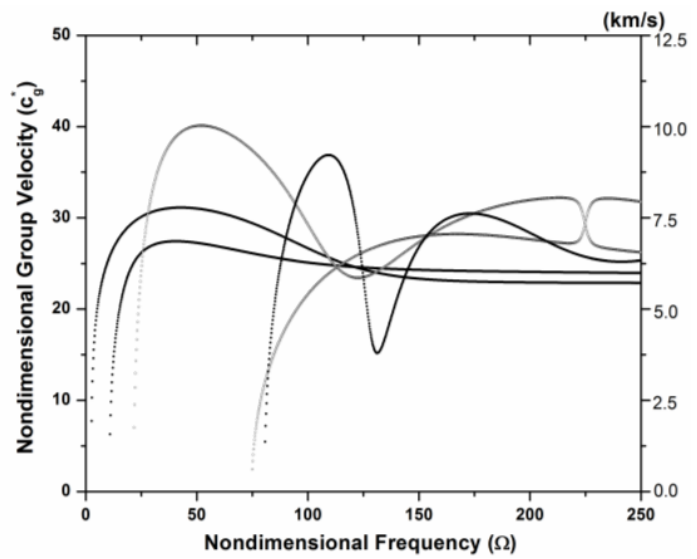

Figure 4. Group velocity of circumferential propagating waves of a silicon nitride cylinder when axial wave number, $m=1$

From the results it is observed that first two modes are almost non-dispersive at high frequencies and approach the Rayleigh wave velocity. It is found that elastic modes are not much affected by the thermal effects within the frequency range considered here. Thermal modes are found to have very high attenuation when compared with elastic modes. Higher order thermal modes originate with high imaginary values of the wave number when $\omega^{*}=0$. It is also found that the higher order thermal modes approach the first thermal mode as the frequency increases.

The dependence of natural frequencies of the finite cylinder on geometry ratio was studied as well by considering different geometry ratio $\left(L^{*}=L / H\right)$ and the material properties of the cylinder. The results will be shown here due to the limitation.

\section{Conclusion}

The natural frequencies of vibration of finite length cylinders have been studied in the context of the L-S generalized theory of thermoelasticity. The proposed SAFE formulation is validated by comparing predicted numerical results with those obtained in earlier investigations. Thermal modes have a much higher attenuation than the elastic modes and the influence of thermal effects on elastic modes is not significant. The guided waves studied in this paper can be used for detecting cracks predominantly in the axial direction in finite cylinders.

\section{References}

1. G.W. McMahon, J. of the Acou. Soc. Am., 36, 85$92(1964)$

2. G.W. McMahon, J. of the Acou. Soc. Am., 48, 307312 (1970)

3. Hutchinson, J.R., J. Acou. Soc. Am., 51, 233240(1972)

4. G.M.L. Gladwell, and U. C. Tahbildar, J. Sound \& Vib, 22, 143-157 (1972)

5. G.M.L. Gladwell, and D.K. Vijay, J. Sound \& Vib, 42, 387-397 (1975)

6. C.P. Lusher, and W.N. Hardy, ASME J. Appl. Mech., 55, 855-862 (1988)

7. R.W. Morse, J. Acou. Soc. Am., 26, 1018-1021 (1954)

8. P. R. Heyliger, J. Sound \& Vib, 148, 507-520 (1991)

9. K.T. Chau, ASME J. Appl. Mech., 61, 964-970 (1994)

10. F. Honarvar, E. Enjilela, and A.N. Sinclair, Acous. Phy., 55, 708-714 (2009)

11. W. Chen, J. Cai, G. Ye, and H. Ding, J. Sound \& Vib, 195, 117-135 (1996)

12. G. Liu, and J. Qu, ASME J. Appl. Mech., 65, 424 430 (1998)

13. M. Venkatesan, and P. Ponnusamy Int. J. Mech. Sci., 49, 741-751 (2007)

14. J. Yu, B. Wu, and C. He, Ultrasonics, 50, 416-423 (2010)

15. S.M. Hosseini, and F. Shahabian, Appl. Math. Modelling, 35, 4697-4714 (2011)

16. H.W. Lord, and Y. Shulman, J. Mech. Phy. Solids, 15, 299-309 (1967)

17. R.B. Nelson, S.B. Dong, and R.D. Kalra, J. Sound \& Vib, 18, 429-444 (1971)

18. K.H. Huang, and S.B. Dong, J. Sound \& Vib, 96(3), 363-379 (1984)

19. S.K. Datta, and A.H. Shah, Elastic Waves in Composite Media and Structures: With Applications to Ultrasonic Nondestructive Evaluation, CRC Press, Boca Raton, (2009)

20. H.M. Al-Qahtani, and S.K. Datta, J. Appl. Phys., 96, 3645-3658 (2004)

21. R. Chitikireddy, Ph.D. Dissertation, University of Manitoba, Winnipeg, MB, Canada, (2012) 